International Journal of Pure and Applied Mathematics

Volume 91 No. 1 2014, 113-129

ISSN: 1311-8080 (printed version); ISSN: 1314-3395 (on-line version)

url: http://www.ijpam.eu

doi: http://dx.doi.org/10.12732/ijpam.v91i1.12

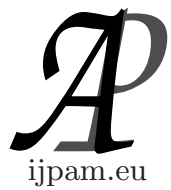

\title{
ALEXANDROV L-TOPOLOGIES AND $L$-JOIN MEET APPROXIMATION OPERATORS
}

\author{
Yong Chan Kim \\ Department of Mathematics \\ Gangneung-Wonju University \\ Gangneung, Gangwondo, 210-702, KOREA
}

\begin{abstract}
In this paper, we investigate the properties of $L$-fuzzy relations and $L$-join meet approximation operators induced by Alexandrov $L$-topologies in complete residuated lattices. We investigate relations among their operations, $L$-fuzzy relations and Alexandrov $L$-topologies.
\end{abstract}

AMS Subject Classification: 03E72, 03G10, 06A15, 06F07

Key Words: complete residuated lattices, $L$-fuzzy preorder, $L$-join meet approximation operators, Alexandrov $L$-topologies

\section{Introduction}

Pawlak $[7,8]$ introduced the rough set theory as a formal tool to deal with imprecision and uncertainty in data analysis. Hájek [2] introduced a complete residuated lattice which is an algebraic structure for many valued logic. Radzikowska [9] developed fuzzy rough sets induced by various $L$-fuzzy relations in complete residuated lattice. Bělohlávek [1] investigated information systems and decision rules in complete residuated lattices. Lai $[5,6]$ introduced Alexandrov $L$-topologies induced by fuzzy rough sets. Algebraic structures of fuzzy rough sets are developed in many directions $[3,4,10,11]$. Kim $[3,4]$ introduced $L$-join meet and $L$-meet join approximation operators as a generalization of fuzzy rough set in complete residuated lattices.

Received: December 27, 2013

(c) 2014 Academic Publications, Ltd. url: www.acadpubl.eu 
In this paper, we investigate the properties of $L$-fuzzy relations and $L$-join meet approximation operators induced by Alexandrov $L$-topologies in complete residuated lattices. We investigate relations among their operations, $L$-fuzzy relations and Alexandrov $L$-topologies.

\section{Preliminaries}

Definition 1. [1,2] An algebra $(L, \wedge, \vee, \odot, \rightarrow, \perp, \top)$ is called a complete residuated lattice if it satisfies the following conditions:

(C1) $L=(L, \leq, \vee, \wedge, \perp, \top)$ is a complete lattice with the greatest element $\top$ and the least element $\perp$;

(C2) $(L, \odot, \top)$ is a commutative monoid;

(C3) $x \odot y \leq z$ iff $x \leq y \rightarrow z$ for $x, y, z \in L$.

In this paper, we assume $\left(L, \wedge, \vee, \odot, \rightarrow,{ }^{*} \perp, \top\right)$ is a complete residuated lattice with the law of double negation;i.e. $x^{* *}=x$. For $\alpha \in L, A, \top_{x} \in L^{X}$, $(\alpha \rightarrow A)(x)=\alpha \rightarrow A(x), \quad(\alpha \odot A)(x)=\alpha \odot A(x)$ and $\top_{x}(x)=\top, \top_{x}(y)=$ $\perp$, otherwise.

Lemma 2. $[1,2]$ For each $x, y, z, x_{i}, y_{i} \in L$, we have the following properties:

(1) If $y \leq z, x \odot y \leq x \odot z, x \rightarrow y \leq x \rightarrow z$ and $z \rightarrow x \leq y \rightarrow x$.

(2) $x \rightarrow\left(\bigwedge_{i \in \Gamma} y_{i}\right)=\bigwedge_{i \in \Gamma}\left(x \rightarrow y_{i}\right)$.

(3) $\left(\bigvee_{i \in \Gamma} x_{i}\right) \rightarrow y=\bigwedge_{i \in \Gamma}\left(x_{i} \rightarrow y\right)$.

(4) $\bigwedge_{i \in \Gamma} y_{i}^{*}=\left(\bigvee_{i \in \Gamma} y_{i}\right)^{*}$ and $\bigvee_{i \in \Gamma} y_{i}^{*}=\left(\bigwedge_{i \in \Gamma} y_{i}\right)^{*}$.

(5) $(x \odot y) \rightarrow z=x \rightarrow(y \rightarrow z)=y \rightarrow(x \rightarrow z)$.

(6) $x \odot y=\left(x \rightarrow y^{*}\right)^{*}$.

(7) $x \odot(x \rightarrow y) \leq y$.

(8) $(x \rightarrow y) \odot(y \rightarrow z) \leq x \rightarrow z$.

Definition 3. [1,5] Let $X$ be a set. A function $R: X \times X \rightarrow L$ is called:

(R1) reflexive if $R(x, x)=\top$ for all $x \in X$,

(R2) symmetric if $R(x, x)=\top$ for all $x \in X$,

(R3) transitive if $R(x, y) \odot R(y, z) \leq R(x, z)$, for all $x, y, z \in X$.

(R4) Euclidean if $R(x, z) \odot R(y, z) \leq R(x, y)$, for all $x, y, z \in X$. 
If $R$ satisfies (R1) and (R3), $R$ is called an $L$-fuzzy preorder.

If $R$ satisfies (R1), (R2) and (R3), $R$ is called an $L$-fuzzy equivalence relation.

Definition 4. [3,4] (1) A map $\mathcal{H}: L^{X} \rightarrow L^{X}$ is called an L-upper approximation operator iff it satisfies the following conditions

(H1) $A \leq \mathcal{H}(A)$,

(H2) $\mathcal{H}(\alpha \odot A)=\alpha \odot \mathcal{H}(A)$ where $\alpha(x)=\alpha$ for all $x \in X$,

(H3) $\mathcal{H}\left(\bigvee_{i \in I} A_{i}\right)=\bigvee_{i \in I} \mathcal{H}\left(A_{i}\right)$

(2) A map $\mathcal{K}: L^{X} \rightarrow L^{X}$ is called an $L$-join meet approximation operator iff it satisfies the following conditions

(K1) $\mathcal{K}(A) \leq A^{*}$,

(K2) $\mathcal{K}(\alpha \odot A)=\alpha \rightarrow \mathcal{K}(A)$,

(K3) $\mathcal{K}\left(\bigvee_{i \in I} A_{i}\right)=\bigwedge_{i \in I} \mathcal{K}\left(A_{i}\right)$.

Definition 5. [3,4] A subset $\tau \subset L^{X}$ is called an Alexandrov L-topology if it satisfies:

(T1) $\perp_{X}, \top_{X} \in \tau$ where $\top_{X}(x)=\top$ and $\perp_{X}(x)=\perp$ for $x \in X$.

(T2) If $A_{i} \in \tau$ for $i \in \Gamma, \bigvee_{i \in \Gamma} A_{i}, \bigwedge_{i \in \Gamma} A_{i} \in \tau$.

(T3) $\alpha \odot A \in \tau$ for all $\alpha \in L$ and $A \in \tau$.

(T4) $\alpha \rightarrow A \in \tau$ for all $\alpha \in L$ and $A \in \tau$.

Theorem 6. $[3,4](1) \tau$ is an Alexandrov $L$-topology on $X$ iff $\tau_{*}=\left\{A^{*} \in\right.$ $\left.L^{X} \mid A \in \tau\right\}$ is an Alexandrov L-topology on $X$.

(2) If $\mathcal{K}$ is an $L$-join meet approximation operator, then $\tau_{\mathcal{K}}=\left\{A \in L^{X}\right.$ $\left.\mathcal{K}(A)=A^{*}\right\}$ is an Alexandrov L-topology on $X$.

(3) If $\mathcal{K}$ is an $L$-join meet approximation operator with $\mathcal{K}\left(\mathcal{K}^{*}(A)\right)=\mathcal{K}(A)$, then $\tau_{\mathcal{K}}=\left\{A \in L^{X} \mid \mathcal{K}(A)=A^{*}\right\}=\left\{\mathcal{K}^{*}(A) \mid A \in L^{X}\right\}$ is an Alexandrov $L$-topology on $X$.

(4) If $\mathcal{K}$ is an $L$-join meet approximation operator with $\mathcal{K}(\mathcal{K}(A))=\mathcal{K}^{*}(A)$, then $\tau_{\mathcal{K}}=\left\{\mathcal{K}(A) \mid A \in L^{X}\right\}=\left(\tau_{\mathcal{K}}\right)_{*}$ is an Alexandrov L-topology on $X$.

Theorem 7. [3] (1) A map $\mathcal{K}: L^{X} \rightarrow L^{X}$ is an $L$-join meet approximation operator iff there exists a reflexive $L$-fuzzy relation $R \in L^{X \times X}$ such that

$$
\mathcal{K}(A)(y)=\bigwedge_{x \in X}\left(A(x) \rightarrow R^{*}(x, y)\right) .
$$


(2) A map $\mathcal{K}: L^{X} \rightarrow L^{X}$ is an $L$-join meet approximation operator with $\mathcal{K}\left(\mathcal{K}^{*}(A)\right)=\mathcal{K}(A)$ iff there exists an $L$-fuzzy preorder $R \in L^{X \times X}$ such that

$$
\mathcal{K}(A)(y)=\bigwedge_{x \in X}\left(A(x) \rightarrow R^{*}(x, y)\right) .
$$

(3) $A \operatorname{map} \mathcal{K}: L^{X} \rightarrow L^{X}$ is an $L$-join meet approximation operator with $\mathcal{K}(\mathcal{K}(A))=\mathcal{K}^{*}(A)$ iff there exists a reflexive and Euclidean $L$-fuzzy relation $R \in L^{X \times X}$ such that

$$
\mathcal{K}(A)(y)=\bigwedge_{x \in X}\left(A(x) \rightarrow R^{*}(x, y)\right) .
$$

Theorem 8. [3] Let $R \in L^{X \times X}$ be a relation. Define operators as follows

$$
\begin{aligned}
\mathcal{K}_{R^{*}}(A)(y) & =\bigwedge_{x \in X}\left(A(x) \rightarrow R^{*}(x, y)\right), \\
\mathcal{K}_{R^{-1 *}}(A)(y) & =\bigwedge_{x \in X}\left(A(x) \rightarrow R^{-1 *}(x, y)\right) .
\end{aligned}
$$

Then the following properties hold.

(1) If $R$ is reflexive, then $\tau_{\mathcal{K}_{R^{*}}}=\tau_{\left(\mathcal{K}_{R^{-1 *}}\right)_{*}}$.

(2) If $R$ is an L-fuzzy preorder, then

$$
\begin{aligned}
\tau_{\mathcal{K}_{R^{*}}} & =\left\{\bigvee_{x \in X}(A(x) \odot R(x,-)) \mid A \in L^{X}\right\} \\
\tau_{\mathcal{K}_{R^{-1 *}}} & =\left\{\bigvee_{x \in X}(A(x) \odot R(-, x)) \mid A \in L^{X}\right\} .
\end{aligned}
$$

(3) If $R$ is reflexive and and Euclidean, then $R$ is symmetric, $R$ is an $L$-fuzzy preorder and $\tau_{\mathcal{K}_{R^{*}}}=\tau_{\mathcal{K}_{R^{-1 *}}}=\tau_{\left(\mathcal{K}_{R^{-1 *}}\right)_{*}}$ such that

$$
\begin{aligned}
\tau_{\mathcal{K}_{R^{*}}} & =\left\{\bigwedge_{x \in X}\left(A(x) \rightarrow R^{*}(x,-)\right) \mid A \in L^{X}\right\} \\
\tau_{\mathcal{K}_{R^{-1 *}}} & =\left\{\bigwedge_{x \in X}\left(A(x) \rightarrow R^{*}(-, x)\right) \mid A \in L^{X}\right\} .
\end{aligned}
$$

\section{Alexandrov $L$-Topologies and $L$-Join Meet Approximation Operators}

Theorem 9. Let $\tau$ be an Alexandrov L-topology on $X$. Then the following properties hold.

(1) There exists an L-fuzzy preorder $R_{\tau}$ such that $\tau=\tau_{\mathcal{K}_{R_{\tau}^{*}}}$ where

$$
\mathcal{K}_{R_{\tau}^{*}}(A)(y)=\bigwedge_{x \in X}\left(A(x) \rightarrow R_{\tau}^{*}(x, y)\right)
$$


(2) Define $\mathcal{H}_{\tau}(A)=\bigwedge\left\{A_{i} \mid A \leq A_{i}, A_{i} \in \tau\right\}$. Then $\mathcal{H}_{\tau}$ is an L-upper approximation operator on $X$ such that $\mathcal{H}_{\tau}=\mathcal{K}_{R_{\tau}^{*}}^{*}$ and $\tau_{\mathcal{H}_{\tau}}=\tau=\tau_{\mathcal{K}_{R_{\tau}^{*}}}$ with

$$
\mathcal{H}_{\tau}(A)(y)=\bigvee_{x \in X}\left(A(x) \odot R_{\tau}(x, y)\right)
$$

(3) Define $k_{\tau}(A)=\bigvee\left\{A_{i} \mid A_{i} \leq A^{*}, A_{i} \in \tau\right\}$. Then $k_{\tau}$ is an $L$-join meet approximation operator on $X$ such that $k_{\tau}\left(k_{\tau}^{*}(A)\right)=k_{\tau}(A)$ for all $A \in L^{X}$. Moreover, $\tau_{k_{\tau}}=\tau_{*}$ and $k_{\tau}(A)=\left(\mathcal{H}_{\tau_{*}}(A)\right)^{*}$ such that

$$
k_{\tau}(A)(y)=\bigwedge_{x \in X}\left(A(x) \rightarrow R_{\tau}^{-1 *}(x, y)\right)
$$

(4) If $R_{\tau}$ is Euclidean, then $k_{\tau}=\mathcal{K}_{R_{\tau}^{*}}$ and $\tau_{*}=\tau_{k_{\tau}}=\tau_{\mathcal{K}_{R_{\tau}^{*}}}=\tau$.

Proof. (1) Define $R_{\tau}(x, y)=\bigwedge_{B \in \tau}(B(x) \rightarrow B(y))$. Then $R_{\tau}(x, x)=\top$ and $R_{\tau}(x, y) \odot R_{\tau}(y, z) \leq R_{\tau}(x, z)$ because $(B(x) \rightarrow B(y)) \odot(B(y) \rightarrow B(z)) \leq$ $(B(x) \rightarrow B(z))$. Let $A \in \tau$.

$$
\begin{aligned}
A(x) \odot R_{\tau}(x, y) & =A(x) \odot \bigwedge_{B \in \tau}(B(x) \rightarrow B(y)) \\
& \leq A(x) \odot(A(x) \rightarrow A(y)) \leq A(y) .
\end{aligned}
$$

Thus $A^{*}(y) \leq\left(A(x) \odot R_{\tau}(x, y)\right)^{*}=A(x) \rightarrow R_{\tau}^{*}(x, y)$. Hence $\mathcal{K}_{R_{\tau}^{*}}(A)=A^{*}$, that is, $A \in \tau_{\mathcal{K}_{R_{\tau}^{*}}}$. Thus $\tau \subset \tau_{\mathcal{K}_{R_{\tau}^{*}}}$.

Let $A \in \tau_{\mathcal{K}_{R_{\tau}^{*}}}$ with $\mathcal{K}_{R_{\tau}^{*}}(A)=A^{*}$. Then $A^{*}(x)=\bigwedge_{y}\left(A(y) \rightarrow R_{\tau}^{*}(y, x)\right)$. So, $A(x)=\bigvee_{y}\left(A(y) \odot R_{\tau}(y, x)\right)=\bigvee_{y}\left(A(y) \odot \bigwedge_{B \in \tau}(B(y) \rightarrow B(x))\right.$. Since $\bigwedge_{B \in \tau}(B(y) \rightarrow B) \in \tau$ from $(\mathrm{T} 4)$ and $\bigvee_{y \in X}\left(A(y) \odot \bigwedge_{B \in \tau}(B(y) \rightarrow B)\right) \in \tau$ from (T3) and (T4), we have $A \in \tau$. Hence $\tau=\tau_{\mathcal{K}_{R_{\tau}^{*}}}$.

(2) Since $R_{\tau}$ is an $L$-fuzzy preorder, by Theorem $7(2), \mathcal{K}_{R_{\tau}^{*}}\left(\mathcal{K}_{R_{\tau}^{*}}^{*}(A)\right)=$ $\mathcal{K}_{R_{\tau}^{*}}(A)$. From the definition of $\mathcal{H}_{\tau}, \mathcal{H}_{\tau}(A) \leq \mathcal{K}_{R_{*}^{*}}^{*}(A)$.

Since $\mathcal{H}_{\tau}(A) \in \tau=\tau_{\mathcal{K}_{R_{\tau}^{*}}}$, we have $\mathcal{K}_{R_{\tau}^{*}}\left(\mathcal{H}_{\tau}(A)\right)=\mathcal{H}_{\tau}^{*}(A)$. Since $\mathcal{K}_{R_{\tau}^{*}}^{*}$ is an increasing function by $(\mathrm{K} 3)$, for $A \leq \mathcal{H}_{\tau}(A)$, we have

$$
\begin{aligned}
\mathcal{K}_{R_{\tau}^{*}}^{*}(A) & \leq \mathcal{K}_{R_{\tau}^{*}}^{*}\left(\mathcal{H}_{\tau}(A)\right)=\mathcal{H}_{\tau}(A) . \\
\mathcal{H}_{\tau}(A)(y) & =\mathcal{K}_{R_{\tau}^{*}}^{*}(A)(y) \\
& =\left(\bigwedge_{x \in X}\left(A(x) \rightarrow R_{\tau}^{*}(x, y)\right)\right)^{*} \\
& =\bigvee_{x \in X}\left(A(x) \odot R_{\tau}(x, y)\right) .
\end{aligned}
$$


Thus, $\mathcal{H}_{\tau}$ is an $L$-upper approximation operator.

(3) (K1) From the definition of $k_{\tau}, k_{\tau}(A) \leq A^{*}$.

(K2) Since $B_{i} \leq \alpha \rightarrow \alpha \odot B_{i}$, we have

$$
\begin{aligned}
k_{\tau}(\alpha \odot A)(x) & =\bigvee\left\{B_{i}(x) \mid B_{i} \leq(\alpha \odot A)^{*}=\alpha \rightarrow A^{*}, B_{i} \in \tau\right\} \\
& \leq \alpha \rightarrow \bigvee\left\{\alpha \odot B_{i}(x) \mid \alpha \odot B_{i} \leq A^{*}, B_{i} \in \tau\right\} \\
& \leq \alpha \rightarrow k_{\tau}(A)(x)
\end{aligned}
$$

Suppose $k_{\tau}(\alpha \odot A) \nsupseteq \alpha \rightarrow k_{\tau}(A)$. Then there exists $x \in X$ such that

$$
k_{\tau}(\alpha \odot A) \gtreqless \alpha \rightarrow k_{\tau}(A) .
$$

From the definition of $k_{\tau}$, there exists $A_{i} \in \tau$ such that $A_{i} \leq A^{*}$ with

$$
k_{\tau}(\alpha \odot A) \nsupseteq \alpha \rightarrow A_{i} .
$$

On the other hand, since $\alpha \rightarrow A_{i} \leq \alpha \rightarrow A^{*}=(\alpha \odot A)^{*}, k_{\tau}(\alpha \odot A) \geq \alpha \rightarrow A_{i}$. It is a contradiction. Thus, $k_{\tau}(\alpha \odot A) \geq \alpha \rightarrow k_{\tau}(A)$. Hence (K2) holds.

(K3) Since $k_{\tau}(B) \leq k_{\tau}(A)$ for $A \leq B$,

$$
k_{\tau}\left(\bigvee_{i \in \Gamma} A_{i}\right) \leq \bigwedge_{i \in \Gamma} k_{\tau}\left(A_{i}\right)
$$

Suppose $k_{\tau}\left(\bigvee_{i \in \Gamma} A_{i}\right) \nsupseteq \bigwedge_{i \in \Gamma} k_{\tau}\left(A_{i}\right)$. From the definition of $k_{\tau}\left(A_{i}\right)$, for all $i \in \Gamma$, there exists $B_{i} \in \tau$ such that $B_{i} \leq A_{i}^{*}$ with

$$
k_{\tau}\left(\bigvee_{i \in \Gamma} A_{i}\right) \nsupseteq \bigwedge_{i \in \Gamma} B_{i} .
$$

On the other hand, since $\bigwedge_{i \in \Gamma} B_{i} \leq \bigwedge_{i \in \Gamma} A_{i}^{*}=\left(\bigvee_{i \in \Gamma} A_{i}\right)^{*}$,

$$
k_{\tau}\left(\bigvee_{i \in \Gamma} A_{i}\right) \geq \bigwedge_{i \in \Gamma} B_{i} .
$$

It is a contradiction. Thus, $k_{\tau}\left(\bigvee_{i \in \Gamma} A_{i}\right) \geq \bigwedge_{i \in \Gamma} k_{\tau}\left(A_{i}\right)$. Hence (K3) holds.

Since $k_{\tau}(A) \in \tau$, by the definition of $k_{\tau}, k_{\tau}(A) \leq k_{\tau}\left(k_{\tau}^{*}(A)\right) \leq k_{\tau}(A)$. Then $k_{\tau}\left(k_{\tau}^{*}(A)\right)=k_{\tau}(A)$.

Let $A \in \tau_{*}$. Then $A^{*} \in \tau$. By the definition of $k_{\tau}, k_{\tau}(A)=A^{*}$. So, $A \in \tau_{k_{\tau}}$. Thus $\tau_{*} \subset \tau_{k_{\tau}}$. Conversely, it similarly proved.

$$
\begin{aligned}
\left(\mathcal{H}_{\tau_{*}}(A)\right)^{*} & =\left(\bigwedge\left\{A_{i} \mid A \leq A_{i}, A_{i} \in \tau_{*}\right\}\right)^{*} \\
& =\bigvee\left\{A_{i}^{*} \mid A_{i}^{*} \leq A^{*}, A_{i}^{*} \in \tau\right\}=k_{\tau}(A) .
\end{aligned}
$$


By (2), since $R_{\tau}^{-1}(x, y)=\bigwedge_{B \in \tau}(B(y) \rightarrow B(x))=\bigwedge_{B \in \tau}\left(B^{*}(x) \rightarrow B^{*}(y)\right)=$ $\bigwedge_{A \in \tau^{*}}(A(x) \rightarrow A(y))$, we have

$$
\begin{aligned}
k_{\tau}(A)(y) & =\left(\mathcal{H}_{\tau_{*}}(A)(y)\right)^{*}=\left(\bigvee_{x \in X}\left(A(x) \odot R_{\tau_{*}}(x, y)\right)\right)^{*} \\
& =\bigwedge_{x \in X}\left(A(x) \rightarrow R_{\tau}^{-1}(x, y)\right) .
\end{aligned}
$$

(4) Since $R_{\tau}$ is Euclidean, then $\bigvee_{x \in X}\left(R_{\tau}(y, x) \odot R_{\tau}(z, x)\right) \leq R_{\tau}(y, z)$. It follows

$$
\begin{aligned}
\mathcal{K}_{R_{\tau}^{*}}\left(\mathcal{K}_{R_{\tau}^{*}}(A)\right)(x) & =\bigwedge_{y \in X}\left(\mathcal{K}_{R_{\tau}^{*}}(A)(y) \rightarrow R_{\tau}^{*}(y, x)\right) \\
& =\bigwedge_{y \in X}\left(\bigwedge_{z \in X}\left(A(z) \rightarrow R_{\tau}^{*}(z, y)\right) \rightarrow R_{\tau}^{*}(y, x)\right) \\
& =\bigwedge_{y \in X}\left(R_{\tau}(y, x) \rightarrow\left(\bigwedge_{z \in X}\left(A(z) \rightarrow R_{\tau}^{*}(z, y)\right)\right)^{*}\right) \\
& =\bigwedge_{y \in X}\left(R_{\tau}(y, x) \rightarrow \bigvee_{z \in X}\left(A(z) \odot R_{\tau}(z, y)\right)\right) \\
& \geq \bigvee_{z \in X}\left(A(z) \odot R_{\tau}(z, x)\right)=\left(\bigwedge_{z \in X}\left(A(z) \rightarrow R_{\tau}(z, x)\right)\right)^{*} \\
& =\left(\mathcal{K}_{R_{\tau}^{*}}(A)\right)^{*}(x) .
\end{aligned}
$$

Thus $\mathcal{K}_{R_{\tau}^{*}}\left(\mathcal{K}_{R_{\tau}^{*}}(A)\right)=\mathcal{K}_{R_{\tau}^{*}}^{*}(A)$ and $\mathcal{K}_{R_{\tau}^{*}}(A) \leq A^{*}$. Since $\tau=\tau_{\mathcal{K}_{R_{\tau}^{*}}}$, by the definition of $k_{\tau}, \mathcal{K}_{R_{\tau}^{*}}(A) \leq k_{\tau}(A)$.

Since $k_{\tau}(A) \leq A^{*}$ iff $A \leq k_{\tau}^{*}(A)=\mathcal{K}_{R_{\tau}^{*}}\left(k_{\tau}(A)\right)$ and $\tau=\tau_{\mathcal{K}_{R_{\tau}^{*}}}$, by the definition of $k_{\tau}$, then

$$
\mathcal{K}_{R_{\tau}^{*}}(A) \geq \mathcal{K}_{R_{\tau}^{*}}\left(\mathcal{K}_{R_{\tau}^{*}}\left(k_{\tau}(A)\right)\right)=\mathcal{K}_{R_{\tau}^{*}}^{*}\left(k_{\tau}(A)\right)=k_{\tau}(A) .
$$

Theorem 10. Let $\tau$ be an Alexandrov L-topology on $X$. Then the following properties hold.

(1) There exists an $L$-fuzzy preorder $R_{\tau}^{-1}$ such that $\tau_{*}=\tau_{\mathcal{K}_{R_{\tau}^{-1 *}}}$ where

$$
\mathcal{K}_{R^{-1 *}}(A)(y)=\bigwedge_{x \in X}\left(A(x) \rightarrow R^{-1 *}(x, y)\right)
$$

(2) Define $\mathcal{H}_{\tau_{*}}(A)=\bigwedge\left\{A_{i} \mid A \leq A_{i}, A_{i} \in \tau_{*}\right\}$. Then $\mathcal{H}_{\tau_{*}}$ is an L-upper approximation operator on $X$ such that $\mathcal{H}_{\tau_{*}}=\mathcal{K}_{R_{\tau}^{-1 *}}^{*}$ and $\tau_{*}=\tau_{\mathcal{H}_{\tau_{*}}}=\tau_{\mathcal{K}_{R_{\tau}^{-1 *}}}$ with

$$
\mathcal{H}_{\tau_{*}}(A)(y)=\bigvee_{x \in X}\left(A(x) \odot R_{\tau}^{-1}(x, y)\right)
$$


(3) Define $k_{\tau_{*}}(A)=\bigvee\left\{A_{i} \mid A_{i} \leq A^{*}, A_{i} \in \tau_{*}\right\}$. Then $k_{\tau_{*}}$ is an $L$-join meet approximation operator on $X k_{\tau_{*}}\left(k_{\tau_{*}}^{*}(A)\right)=k_{\tau_{*}}(A)$ for all $A \in L^{X}$. Moreover, $\tau_{k_{\tau_{*}}}=\tau$ and $k_{\tau_{*}}(A)=\left(\mathcal{H}_{\tau}(A)\right)^{*}$ such that

$$
k_{\tau_{*}}(A)(y)=\bigwedge_{x \in X}\left(A(x) \rightarrow R_{\tau}^{*}(x, y)\right) .
$$

(4) If $R_{\tau}^{-1}$ is Euclidean, then $k_{\tau_{*}}=\mathcal{K}_{R_{\tau}^{-1 *}}$ and $\tau=\tau_{k_{*}}=\tau_{\mathcal{K}_{R_{\tau}^{-1 *}}}=\tau_{*}$.

Proof. (1) Define $R_{\tau}^{-1}(x, y)=\bigwedge_{B \in \tau}(B(y) \rightarrow B(x))=\bigwedge_{B \in \tau}\left(B^{*}(x) \rightarrow\right.$ $\left.B^{*}(y)\right)$. Then $R_{\tau}^{-1}(x, x)=\top$ and $R_{\tau}^{-1}(x, y) \odot R_{\tau}^{-1}(y, z) \leq R_{\tau}^{-1}(x, z)$. Let $A^{*} \in \tau_{*}$.

$$
\begin{aligned}
A^{*}(x) \odot R_{\tau}^{-1}(x, y) & =A^{*}(x) \odot \bigwedge_{B \in \tau}\left(B^{*}(x) \rightarrow B^{*}(y)\right) \\
& \leq A^{*}(x) \odot\left(A^{*}(x) \rightarrow A^{*}(y)\right) \leq A^{*}(y) .
\end{aligned}
$$

Thus $A(y) \leq\left(A^{*}(x) \odot R_{\tau}^{-1}(x, y)\right)^{*}=A^{*}(x) \rightarrow R_{\tau}^{-1 *}(x, y)$. Hence $\mathcal{K}_{R_{\tau}^{-1 *}}\left(A^{*}\right)=$ $A$, that is, $A^{*} \in \tau_{\mathcal{K}_{R_{\tau}^{-1 *}}}$.

Let $A \in \tau_{\mathcal{K}_{R_{\tau}^{-1 *}}}$ with $\mathcal{K}_{R_{\tau}^{-1 *}}(A)=A^{*}$. Then $A^{*}(x)=\bigwedge_{y}\left(A(y) \rightarrow R_{\tau}^{-1 *}(y, x)\right)$. So, $A(x)=\bigvee_{y}\left(A(y) \odot R_{\tau}^{-1}(y, x)\right)=\bigvee_{y}\left(A(y) \odot \bigwedge_{B \in \tau}(B(x) \rightarrow B(y))=\bigvee_{y}(A(y) \odot\right.$ $\bigwedge_{B \in \tau}\left(B^{*}(y) \rightarrow B^{*}(x)\right)$. Since $\bigwedge_{B \in \tau}\left(B^{*}(y) \rightarrow B^{*}\right) \in \tau_{*}$ and $\bigvee_{y \in X}(A(y) \odot$ $\left.\bigwedge_{B \in \tau}\left(B^{*}(y) \rightarrow B^{*}\right)\right) \in \tau_{*}$, we have $A \in \tau_{*}$. Hence $\tau_{*}=\tau_{\mathcal{K}_{R_{\tau}^{-1 *}}}$.

(2) and (3) are similarly proved as Theorem 9 (2) and (3), respectively.

(4) Since $R^{-1}$ is Euclidean, then $\bigvee_{x \in X}\left(R_{\tau}(x, y) \odot R_{\tau}(x, z) \leq R_{\tau}(y, z)\right.$. Thus

$$
\begin{aligned}
\mathcal{K}_{R_{\tau}^{-1 *}}\left(\mathcal{K}_{R_{\tau}^{-1 *}}(A)\right)(x) & =\bigwedge_{y \in X}\left(\mathcal{K}_{R_{\tau}^{-1 *}}(A)(y) \rightarrow R_{\tau}^{-1 *}(y, x)\right) \\
& =\bigwedge_{y \in X}\left(\bigwedge_{z \in X}\left(A(z) \rightarrow R_{\tau}^{-1 *}(z, y)\right) \rightarrow R_{\tau}^{-1 *}(y, x)\right) \\
& =\bigwedge_{y \in X}\left(R_{\tau}^{-1}(y, x) \rightarrow\left(\bigwedge_{z \in X}\left(A(z) \rightarrow R_{\tau}^{-1 *}(z, y)\right)\right)^{*}\right) \\
& =\bigwedge_{y \in X}\left(R_{\tau}^{-1}(y, x) \rightarrow \bigvee_{z \in X}\left(A(z) \odot R_{\tau}^{-1}(z, y)\right)\right) \\
& \geq \bigvee_{z \in X}\left(A(z) \odot R_{\tau}^{-1}(z, x)\right)
\end{aligned}
$$

Since $\mathcal{K}_{R_{\tau}^{-1 *}}\left(\mathcal{K}_{R_{\tau}^{-1 *}}(A)\right)=\mathcal{K}_{R_{\tau}^{-1 *}}^{*}(A), \mathcal{K}_{R_{\tau}^{-1 *}}(A) \in \tau_{*}$ and $\mathcal{K}_{R_{\tau}^{-1 *}}(A) \leq A^{*}$, then $\mathcal{K}_{R_{\tau}^{-1 *}}(A) \leq k_{\tau_{*}}(A)$.

Since $k_{\tau_{*}}(A) \leq A^{*}$ iff $A \leq k_{\tau_{*}}^{*}(A)=\mathcal{K}_{R_{\tau}^{-1 *}}\left(k_{\tau_{*}}(A)\right)$ because $k_{\tau_{*}}(A) \in \tau_{*}=$ $\tau_{\mathcal{K}_{R_{\tau}^{-1 *}}}$, then

$$
\mathcal{K}_{R_{\tau}^{-1 *}}(A) \geq \mathcal{K}_{R_{\tau}^{-1 *}}\left(\mathcal{K}_{R_{\tau}}^{-1 *}\left(k_{\tau_{*}}(A)\right)\right)=\mathcal{K}_{R_{\tau}^{-1 *}}^{*}\left(k_{\tau_{*}}(A)\right)=k_{\tau_{*}}(A) .
$$


Theorem 11. Let $\mathcal{K}_{R^{*}}: L^{X} \rightarrow L^{X}$ be an $L$-join meet approximation operator on $X$ defined as

$$
\mathcal{K}_{R^{*}}(A)(y)=\bigwedge_{x \in X}\left(A(x) \rightarrow R^{*}(x, y)\right) .
$$

(1) $A^{*} \leq \mathcal{K}_{R^{*}}(A)$ iff $A \leq \mathcal{K}_{R^{-1 *}}\left(A^{*}\right)$ and $\tau_{\mathcal{K}_{R^{-1 *}}}=\left(\tau_{\mathcal{K}_{R^{*}}}\right)_{*}$.

(2) If $R$ is an $L$-fuzzy preorder and we define $\mathcal{H}_{\tau_{\mathcal{K}_{R^{*}}}}(A)=\bigwedge\left\{A_{i} \mid A \leq\right.$ $\left.A_{i}, A_{i} \in \tau_{\mathcal{K}_{R^{*}}}\right\}$ and $\mathcal{H}_{\tau_{\mathcal{K}_{R^{-1 *}}}}(A)=\bigwedge\left\{A_{i} \mid A \leq A_{i}, A_{i} \in \tau_{\mathcal{K}_{R^{-1 *}}}=\left(\tau_{\mathcal{K}_{R^{*}}}\right)_{*}\right\}$, then $\mathcal{H}_{\tau_{\mathcal{K}_{R^{*}}}}$ and $\mathcal{H}_{\tau_{\mathcal{K}_{R^{-1 *}}}}$ are $L$-upper approximation operators on $X$ such that $\mathcal{H}_{\tau_{\mathcal{K}_{R^{*}}}}=\mathcal{K}_{R^{*}}^{*}$ and $\mathcal{H}_{\tau_{\mathcal{K}_{R^{-1 *}}}}=\mathcal{K}_{R^{-1 *}}^{*}$ with

$$
\begin{aligned}
& \mathcal{H}_{\tau_{\mathcal{K}_{R^{*}}}}(A)(y)=\bigvee_{x \in X}(A(x) \odot R(x, y)) \\
& \mathcal{H}_{\left(\mathcal{K}_{R^{*}}\right)_{*}}(A)(y)=\mathcal{H}_{\tau_{\mathcal{K}_{R^{-1 *}}}}(A)(y)=\bigvee_{x \in X}\left(A(x) \odot R^{-1}(x, y)\right)
\end{aligned}
$$

(3) Define $k_{\tau_{\mathcal{K}_{R^{*}}}}(A)=\bigvee\left\{A_{i} \mid A_{i} \leq A^{*}, A_{i} \in \tau_{\mathcal{K}_{R^{*}}}\right\}$. Then $k_{\tau_{\mathcal{K}_{R^{*}}}}$ is an $L$-join meet approximation operator on $X$ such that $k_{\tau_{\mathcal{K}_{R^{*}}}}\left(k_{\tau_{\mathcal{K}_{R^{*}}}^{*}}(A)\right)=$ $k_{\tau_{\mathcal{K}_{R^{*}}}}(A)$ for all $A \in L^{X}$. Moreover, $\tau_{k_{\tau_{\mathcal{K}_{R^{*}}}}}=\left(\tau_{\mathcal{K}_{R^{*}}}\right)_{*}=\tau_{\mathcal{K}_{R^{-1 *}}}$ and $k_{\tau_{\mathcal{K}_{R^{*}}}}(A)=$ $\left(\mathcal{H}_{\left(\mathcal{K}_{R^{*}}\right)_{*}}(A)\right)^{*}$ such that

$$
k_{\tau_{\mathcal{K}_{R^{*}}}}(A)(y)=\bigwedge_{x \in X}\left(A(x) \rightarrow R^{-1 *}(x, y)\right)
$$

(4) Define $k_{\tau_{\mathcal{K}_{R^{-1 *}}}}(A)=\bigvee\left\{A_{i} \mid A_{i} \leq A^{*}, A_{i} \in \tau_{\mathcal{K}_{R^{-1 *}}}\right\}$. Then $k_{\tau_{\mathcal{K}^{-1 *}}}$ is an $L$-join meet approximation operator on $X$ such that $k_{\tau_{\mathcal{K}_{R^{-1 *}}}}\left(k_{\tau_{\mathcal{K}^{-1 *}}}^{*}(A)\right)=$ $k_{\tau_{\mathcal{K}_{R^{-1 *}}}}(A)$ for all $A \in L^{X}$. Moreover, $\tau_{k_{\tau_{\mathcal{K}}-1 *}}=\left(\tau_{\mathcal{K}_{R^{-1 *}}}\right)_{*}=\tau_{\mathcal{K}_{R^{*}}}$ and $k_{\tau_{\mathcal{K}^{-1 *}}}(A)=\left(\mathcal{H}_{\left(\tau_{\mathcal{K}_{R^{-1 *}}}\right) *}(A)\right)^{*}$ such that

$$
k_{\tau_{\mathcal{K}_{R^{-1 *}}}}(A)(y)=\bigwedge_{x \in X}\left(A(x) \rightarrow R^{*}(x, y)\right) .
$$

(5) If $R$ is Euclidean, then $k_{\tau_{\mathcal{K}_{R^{*}}}}=\mathcal{K}_{R^{*}}$. 
Proof. (1) Since $A^{*}(y) \leq \mathcal{K}_{R^{*}}(A)(y)=\mathcal{K}_{R^{*}}(A)(y)=\bigwedge_{x \in X}\left(A(x) \rightarrow R^{*}(x, y)\right)$ iff $A(x) \leq \bigwedge_{y \in X}\left(A^{*}(y) \rightarrow R^{*}(x, y)\right)=\mathcal{K}_{R^{-1 *}}(A)(x)$, we have $\tau_{\mathcal{K}_{R^{-1 *}}}=\left(\tau_{\mathcal{K}_{R^{*}}}\right)_{*}$.

(2) Since $R$ is an $L$-fuzzy preorder, $\mathcal{K}_{R^{*}}\left(\mathcal{K}_{R^{*}}^{*}(A)\right)=\mathcal{K}_{R^{*}}(A)$. From the definition of $\mathcal{H}_{\tau_{\mathcal{K}_{R^{*}}}}, \mathcal{H}_{\tau_{\mathcal{K}_{R^{*}}}}(A) \leq \mathcal{K}_{R^{*}}^{*}(A)$.

Since $\mathcal{H}_{\tau_{\mathcal{K}_{R^{*}}}}(A) \in \tau_{\mathcal{K}_{R^{*}}}$, we have $\mathcal{K}_{R^{*}}\left(\mathcal{H}_{\tau_{\mathcal{K}_{R^{*}}}}(A)\right)=\mathcal{H}_{\tau_{\mathcal{K}_{R^{*}}}^{*}}(A)$. Since $\mathcal{K}_{R^{*}}^{*}$ is an increasing function by (K3), for $A \leq \mathcal{H}_{\tau_{\mathcal{K}_{R^{*}}}}(A)$, we have

$$
\begin{aligned}
& \mathcal{K}_{R^{*}}^{*}(A) \leq \mathcal{K}_{R^{*}}^{*}\left(\mathcal{H}_{\tau_{\mathcal{K}_{R^{*}}}}(A)\right)=\mathcal{H}_{\tau_{\mathcal{K}_{R^{*}}}}(A) . \\
& \mathcal{H}_{\tau_{\mathcal{K}_{R^{*}}}}(A)(y)=\mathcal{K}_{R^{*}}^{*}(A)(y) \\
&=\left(\bigwedge_{x \in X}\left(A(x) \rightarrow R^{*}(x, y)\right)\right)^{*} \\
&=\bigvee_{x \in X}(A(x) \odot R(x, y)) .
\end{aligned}
$$

Thus, $\mathcal{H}_{\tau_{\mathcal{K}_{R^{*}}}}$ is an $L$-upper approximation operator.

(3) By Theorem $9(3), k_{\tau_{\mathcal{K}_{R^{*}}}}$ is an $L$-join meet approximation operator on $X$. Let $A \in\left(\tau_{\mathcal{K}_{R^{*}}}\right)_{*}$. Then $A^{*} \in \tau_{\mathcal{K}_{R^{*}}}$. By the definition of $k_{\tau_{\mathcal{K}_{R^{*}}}}, k_{\tau_{\mathcal{K}_{R^{*}}}}(A)=A^{*}$.

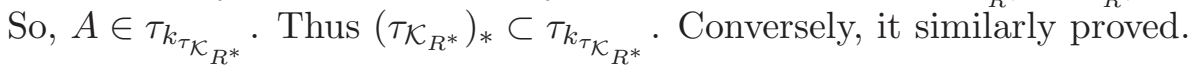

$$
\begin{aligned}
\left(\mathcal{H}_{\left(\tau_{\mathcal{K}^{*}}\right)_{*}}(A)\right)^{*} & =\left(\bigwedge\left\{A_{i} \mid A \leq A_{i}, A_{i} \in\left(\tau_{\mathcal{K}_{R^{*}}}\right)_{*}\right\}\right)^{*} \\
& =\bigvee\left\{A_{i}^{*} \mid A_{i}^{*} \leq A^{*}, A_{i}^{*} \in \tau_{\mathcal{K}_{R^{*}}}\right\}=k_{\tau_{\mathcal{K}_{R^{*}}}}(A) .
\end{aligned}
$$

By (1) an (2), since $\tau_{\mathcal{K}_{R^{-1 *}}}=\left(\tau_{\mathcal{K}_{R^{*}}}\right)_{*}$. and $\mathcal{H}_{\tau_{\mathcal{K}_{R^{-1 *}}}}=\mathcal{K}_{R^{-1 *}}^{*}$, we have

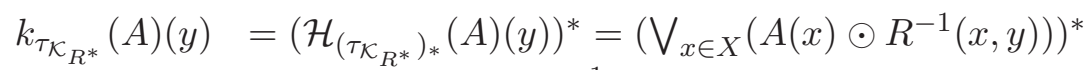

$$
\begin{aligned}
& =\bigwedge_{x \in X}\left(A(x) \rightarrow R^{-1}(x, y)\right) \text {. }
\end{aligned}
$$

(4) It is similarly proved as (3).

(5) Since $R$ is Euclidean, $\bigvee_{x \in X}(R(y, x) \odot R(z, x)) \leq R(y, z)$. By Theorem $7(3), \mathcal{K}_{R^{*}}\left(\mathcal{K}_{R^{*}}(A)\right)=\mathcal{K}_{R^{*}}^{*}(A)$ and $\mathcal{K}_{R^{*}}(A) \leq A^{*}$. By the definition of $k_{\tau_{\mathcal{K}_{R^{*}}}}$, $\mathcal{K}_{R^{*}}(A) \leq k_{\tau_{\mathcal{K}_{R^{*}}}}(A)$. Since $k_{\tau_{\mathcal{K}_{R^{*}}}}(A) \leq A^{*}$ iff $A \leq k_{\tau_{\mathcal{K}_{R^{*}}}}^{*}(A)=\mathcal{K}_{R^{*}}\left(k_{\tau_{\mathcal{K}_{R^{*}}}}(A)\right)$, by the definition of $k_{\tau_{\mathcal{K}_{R^{*}}}}$, then

$$
\mathcal{K}_{R^{*}}(A) \geq \mathcal{K}_{R^{*}}\left(\mathcal{K}_{R^{*}}\left(k_{\tau_{\mathcal{K}_{R^{*}}}}(A)\right)\right)=\mathcal{K}_{R^{*}}^{*}\left(k_{\tau_{\mathcal{K}_{R^{*}}}}(A)\right)=k_{\tau_{\mathcal{K}_{R^{*}}}}(A) .
$$


Theorem 12. Let $R \in L^{X \times X}$ be an $L$-fuzzy relation and $\tau_{\mathcal{K}_{R^{*}}}$ the Alexandrov $L$-topology induced by $\mathcal{K}_{R^{*}}$. Then the following properties hold.

(1) If $R$ is transitive, then $R \leq R_{\mathcal{T}_{R^{*}}}$.

(2) If $R_{z} \in \tau_{\mathcal{K}_{R^{*}}}$ for $z \in X$ where $R_{z}(x)=R(z, x)$ and $\bigwedge_{z \in X}(R(z, x) \rightarrow$ $R(z, y)) \leq R(x, y)$, then $R_{\tau_{\mathcal{K}_{R^{*}}}} \leq R$.

(3) If $R$ is an $L$-fuzzy preorder, then $R=R_{\tau_{\mathcal{K}_{R^{*}}}}$.

(4) If $R$ is Euclidean, then $R^{-1} \leq R_{\tau_{\mathcal{K}_{R^{*}}}}$.

(5) If $R_{z}^{*} \in \tau_{\mathcal{K}_{R^{*}}}$ for $z \in X$ where $R_{z}^{*}(x)=R^{*}(z, x)$ and $\bigwedge_{z \in X}(R(z, x) \rightarrow$ $R(z, y)) \leq R(x, y)$, then $R_{\tau_{\mathcal{K}_{R^{*}}}} \leq R^{-1}$.

(6) If $R$ is reflexive and $R$ is Euclidean, then $R^{-1}=R_{\tau_{\mathcal{K}_{R^{*}}}}$.

(7) If $R$ is an $L$-fuzzy preorder, then $R^{-1}=R_{\tau_{\left(\mathcal{K}_{\left.R^{*}\right) *}\right.}}$.

(8) If $R$ is reflexive and $R$ is Euclidean, then $R=R_{\tau_{\left(\mathcal{K}_{\left.R^{*}\right) *}\right.}}$.

Proof. (1) Since $R(x, y) \odot B(z) \odot R(z, x) \leq B(z) \odot R(z, y)$ iff $R(x, y) \leq$ $B(z) \odot R(z, x) \rightarrow B(z) \odot R(z, y)$. Thus

$$
\begin{aligned}
R_{\tau_{\mathcal{K}_{R^{*}}}}(x, y) & =\bigwedge_{B \in \tau_{\mathcal{K}_{R^{*}}}}(B(x) \rightarrow B(y)) \\
& =\bigwedge_{B \in \tau_{\mathcal{K}_{R^{*}}}}\left(\left(\mathcal{K}_{R^{*}}(B)\right)^{*}(x) \rightarrow\left(\mathcal{K}_{R^{*}}(B)\right)^{*}(y)\right) \\
& \geq \bigwedge_{B \in \tau_{\mathcal{K}_{R^{*}}}} \bigvee_{z \in X}(B(z) \odot R(z, x) \rightarrow B(z) \odot R(z, y)) \\
& \geq R(x, y) .
\end{aligned}
$$

(2) Since $R_{z} \in \tau_{\mathcal{K}_{R^{*}}}$ for $z \in X$, then

$$
\begin{aligned}
R_{\tau_{\mathcal{K}_{R^{*}}}}(x, y) & =\bigwedge_{B \in \tau_{\mathcal{K}_{R^{*}}}}(B(x) \rightarrow B(y)) \\
& \leq \bigwedge_{z \in X}\left(R_{z}(x) \rightarrow R_{z}(y)\right) \\
& =\bigwedge_{z \in X}(R(z, x) \rightarrow R(z, y)) \leq R(x, y)
\end{aligned}
$$

(3) Since $R$ is transitive, by (1), $R \leq R_{\tau_{\mathcal{K}_{R^{*}}}}$. Since $R$ is an $L$-fuzzy preorder, we have $\mathcal{K}_{R^{*}}\left(\mathcal{K}_{R^{*}}^{*}(A)\right)=\mathcal{K}_{R^{*}}(A)$. Since $\mathcal{K}_{R^{*}}^{*}\left(\top_{z}\right)(x)=R(z, x)$, we have

$$
\begin{aligned}
R_{\tau_{\mathcal{K}_{R^{*}}}}(x, y) & =\bigwedge_{B \in \tau_{\mathcal{K}_{R^{*}}}}(B(x) \rightarrow B(y)) \\
& \leq \bigwedge_{z \in X}\left(\mathcal{K}_{R^{*}}^{*}\left(\top_{z}\right)(x) \rightarrow \mathcal{K}_{R^{*}}^{*}\left(\top_{z}\right)(y)\right) \\
& =\bigwedge_{z \in X}(R(z, x) \rightarrow R(z, y)) \leq R(x, x) \rightarrow R(x, y)=R(x, y)
\end{aligned}
$$


(4) Since $R$ is Euclidean, then $R(y, x) \odot B(z) \odot R(z, x) \leq B(z) \odot R(z, y)$ iff $R(y, x) \leq B(z) \odot R(z, x) \rightarrow B(z) \odot R(z, y)$. Thus

$$
\begin{aligned}
R_{\tau_{\mathcal{K}_{R^{*}}}}(x, y) & =\bigwedge_{B \in \tau_{\mathcal{K}_{R^{*}}}}(B(x) \rightarrow B(y)) \\
& =\bigwedge_{B \in \tau_{\mathcal{K}_{R^{*}}}}\left(\left(\mathcal{K}_{R^{*}}(B)\right)^{*}(x) \rightarrow\left(\mathcal{K}_{R^{*}}(B)\right)^{*}(y)\right) \\
& \geq \bigwedge_{B \in \tau_{\mathcal{K}_{R^{*}}}} \bigvee_{z \in X}(B(z) \odot R(z, x) \rightarrow B(z) \odot R(z, y)) \\
& \geq R^{-1}(x, y)
\end{aligned}
$$

(5) Since $R_{z}^{*} \in \tau_{\mathcal{K}_{R^{*}}}$, we have

$$
\begin{aligned}
R_{\tau_{\mathcal{K}_{R^{*}}}}(x, y) & =\bigwedge_{B \in \tau_{\mathcal{K}_{R^{*}}}}(B(x) \rightarrow B(y)) \\
& \leq \bigwedge_{z \in X}\left(R^{*}(z, x) \rightarrow R^{*}(z, y)\right) \\
& =\bigwedge_{z \in X}(R(z, y) \rightarrow R(z, x)) \leq R(y, x)=R^{-1}(x, y)
\end{aligned}
$$

(6) Since $R$ is Euclidean, by (4), $R^{-1} \leq R_{\tau_{\mathcal{K}_{R^{*}}}}$. Since $R$ is reflexive and Euclidean, by Theorem $7(3)$, we have $\mathcal{K}_{R^{*}}\left(\mathcal{K}_{R^{*}}(A)\right)=\mathcal{K}_{R^{*}}^{*}(A)$. Since $\mathcal{K}_{R^{*}}\left(\top_{z}\right)=R^{*}(z,-) \in \tau_{\mathcal{K}_{R^{*}}}$, we have

$$
\begin{aligned}
R_{\tau_{\mathcal{K}_{R^{*}}}}(x, y) & =\bigwedge_{B \in \tau_{\mathcal{K}_{R^{*}}}}(B(x) \rightarrow B(y)) \\
& \leq \bigwedge_{z \in X}\left(\mathcal{K}_{R^{*}}\left(\top_{z}\right)(x) \rightarrow \mathcal{K}_{R^{*}}\left(\top_{z}\right)(y)\right) \\
& =\bigwedge_{z \in X}\left(R^{*}(z, x) \rightarrow R^{*}(z, y)\right)=\bigwedge_{z \in X}(R(z, y) \rightarrow R(z, x)) \\
& \leq R(y, y) \rightarrow R(y, x)=R^{-1}(x, y) .
\end{aligned}
$$

(7) Since $R$ is transitive, we have

$$
\begin{aligned}
R_{\left(\tau_{\left.\mathcal{K}_{R^{*}}\right)_{*}}(x, y)\right.} & =\bigwedge_{B \in\left(\tau_{\left.\mathcal{K}_{R^{*}}\right)_{*}}\right.}(B(x) \rightarrow B(y))=\bigwedge_{B \in \tau_{\mathcal{K}_{R^{*}}}}\left(B^{*}(x) \rightarrow B^{*}(y)\right) \\
& =\bigwedge_{B \in \tau_{\mathcal{K}_{R^{*}}}}\left(\mathcal{K}_{R^{*}}(B)(x) \rightarrow \mathcal{K}_{R^{*}}(B)(y)\right) \\
& \geq \bigwedge_{B \in \tau_{\mathcal{K}_{R^{*}}}} \bigwedge_{z \in X}\left(\left(B(z) \rightarrow R^{*}(z, x)\right) \rightarrow\left(B(z) \rightarrow R^{*}(z, y)\right)\right) \\
& \geq \bigwedge_{z \in X}\left(R^{*}(z, x) \rightarrow R^{*}(z, y)\right)=\bigwedge_{z \in X}(R(z, y) \rightarrow R(z, x)) \\
& \geq R^{-1}(x, y) .
\end{aligned}
$$

Since $R$ is an $L$-fuzzy preorder, by Theorem $7(2), \mathcal{K}_{R^{*}}\left(\mathcal{K}_{R^{*}}^{*}(A)\right)=\mathcal{K}_{R^{*}}(A)$ for all $A \in L^{X}, \mathcal{K}_{R^{*}}\left(\mathcal{K}_{R^{*}}^{*}\left(\top_{z}\right)\right)=\mathcal{K}_{R^{*}}\left(\top_{z}\right)$ for all $z \in X$. So, $\mathcal{K}_{R^{*}}^{*}\left(\top_{z}\right) \in \tau_{\mathcal{K}_{R^{*}}}$. Thus,

$$
\begin{aligned}
R_{\left(\tau_{\left.\mathcal{K}_{R^{*}}\right)^{*}}(x, y)\right.} & =\bigwedge_{B \in \tau_{\mathcal{K}_{R^{*}}}}\left(B^{*}(x) \rightarrow B^{*}(y)\right) \\
& \leq \bigwedge_{z \in X}\left(\mathcal{K}_{R^{*}}\left(\top_{z}\right)(x) \rightarrow \mathcal{K}_{R^{*}}\left(\top_{z}\right)(y)\right) \\
& =\bigwedge_{z \in X}\left(R^{*}(z, x) \rightarrow R^{*}(z, y)\right)=\bigwedge_{z \in X}(R(z, y) \rightarrow R(z, x)) \\
& \leq R(y, y) \rightarrow R(y, x)=R^{-1}(x, y) .
\end{aligned}
$$


(8) Since $\bigvee_{x \in X}(R(z, y) \odot R(x, y)) \leq R(z, x)$ for all $x, y, z \in X$, then $R(z, y) \odot$ $R(x, y) \leq R(z, x)$ iff $R(x, y) \leq R(z, y) \rightarrow R(z, x)$. Thus

$$
\begin{aligned}
R_{\left(\tau_{\left.\mathcal{K}_{R^{*}}\right)_{*}}(x, y)\right.} & =\bigwedge_{B \in\left(\tau_{\left.\mathcal{K}_{R^{*}}\right)_{*}}\right.}(B(x) \rightarrow B(y))=\bigwedge_{B \in \mathcal{T}_{R^{*}}}\left(B^{*}(x) \rightarrow B^{*}(y)\right) \\
& =\bigwedge_{B \in \mathcal{T}_{R^{*}}}\left(\mathcal{K}_{R^{*}}(B)(x) \rightarrow \mathcal{K}_{R^{*}}(B)(y)\right) \\
& \geq \bigwedge_{B \in \tau_{\mathcal{K}_{R^{*}}}}\left(\bigwedge_{z \in X}\left(B(z) \rightarrow R^{*}(z, x)\right) \rightarrow \bigwedge_{w \in X}\left(B(w) \rightarrow R^{*}(w, y)\right)\right) \\
& \geq \bigwedge_{B \in \tau_{\mathcal{K}^{*}}} \bigwedge_{z \in X}\left(\left(B(z) \rightarrow R^{*}(z, x)\right) \rightarrow\left(B(z) \rightarrow R^{*}(z, y)\right)\right) \\
& \geq \bigwedge_{z \in X}\left(R^{*}(z, x) \rightarrow R^{*}(z, y)\right) \\
& =\bigwedge_{z \in X}(R(z, y) \rightarrow R(z, x)) \geq R(x, y)
\end{aligned}
$$

Since $R$ is reflexive and Euclidean, by Theorem $7(3), \mathcal{K}_{R^{*}}\left(\mathcal{K}_{R^{*}}(A)\right)=$ $\mathcal{K}_{R^{*}}^{*}(A)$ for all $A \in L^{X}, \mathcal{K}_{R^{*}}\left(\mathcal{K}_{R^{*}}\left(\top_{z}\right)\right)=\mathcal{K}_{R^{*}}^{*}\left(\top_{z}\right)$ for all $z \in X$. So, $\mathcal{K}_{R^{*}}\left(\top_{z}\right) \in$ $\tau_{\mathcal{K}_{R^{*}}}$. Thus,

$$
\begin{aligned}
R_{\left(\tau_{\mathcal{K}_{R^{*}}}\right)_{*}}(x, y) & =\bigwedge_{B \in \tau_{\mathcal{K}_{R^{*}}}}\left(B^{*}(x) \rightarrow B^{*}(y)\right) \\
& \leq \bigwedge_{z \in X}\left(\mathcal{K}_{R^{*}}^{*}\left(\top_{z}\right)(x) \rightarrow \mathcal{K}_{R^{*}}^{*}\left(\top_{z}\right)(y)\right) \\
& =\bigwedge_{z \in X}(R(z, x) \rightarrow R(z, y)) \leq R(x, x) \rightarrow R(x, y)=R(x, y)
\end{aligned}
$$

Example 13. Let $\left(L=[0,1], \odot, \rightarrow,{ }^{*}\right)$ be a complete residuated lattice with the law of double negation defined by

$$
x \odot y=(x+y-1) \vee 0, x \rightarrow y=(1-x+y) \wedge 1, x^{*}=1-x .
$$

Let $X=\{a, b, c\}$ and $A \in L^{X}$ as follows:

$$
A(a)=1, A(b)=0.1, A(c)=0.4 .
$$

Define $R \in L^{X \times X}$ as follows

$$
R=\left(\begin{array}{ccc}
1 & 0.2 & 0.9 \\
0.8 & 1 & 0.7 \\
0.6 & 0.5 & 1
\end{array}\right)
$$

(1) Since $0.4=R(a, c) \odot R(c, b) \not \leq R(a, b)=0.2$ and $\mathcal{K}_{R^{*}}(A)=(0,0.8,0.1)$, we have

$$
\mathcal{K}_{R^{*}}(A)=(0,0.8,0.1) \neq \mathcal{K}_{R^{*}}\left(\mathcal{K}_{R^{*}}^{*}(A)\right)=(0,0.6,0.1) .
$$

For $R_{x}=(1,0.2,0.9), R_{y}=(0.8,1,0.7), R_{z}=(0.6,0.5,1)$, since

$$
\mathcal{K}_{R^{*}}\left(R_{x}\right)=(0,0.6,0.1) \neq R_{x}^{*}, \quad \mathcal{K}_{R^{*}}\left(R_{y}\right)=(0.2,0,0.3)=R_{y}^{*}
$$




$$
\mathcal{K}_{R^{*}}\left(R_{z}\right)=(0.4,0.5,0)=R_{z}^{*},
$$

Hence $R_{y}, R_{z} \in \tau_{\mathcal{K}_{R^{*}}}$ but $R_{x} \notin \tau_{\mathcal{K}_{R^{*}}}$ and $\bigwedge_{z \in X}(R(z, c) \rightarrow R(z, b))=0.3 \neq$ $R(c, b)=0.5$ from:

$$
\left(\bigwedge_{z \in X}(R(z, x) \rightarrow R(z, y))\right)=\left(\begin{array}{ccc}
1 & 0.2 & 0.9 \\
0.8 & 1 & 0.7 \\
0.6 & 0.3 & 1
\end{array}\right)
$$

(2) Since $0.6=R(a, c) \odot R(b, c) \not \leq R(a, b)=0.2, R$ is not Euclidean. For $\mathcal{K}_{R^{*}}(A)=(0,0.8,0.1)$, we have

$$
\mathcal{K}_{R^{*}}^{*}(A)=(1,0.2,0.9) \neq \mathcal{K}_{R^{*}}\left(\mathcal{K}_{R^{*}}(A)\right)=(0.4,0.2,0.5) .
$$

For $R_{x}^{*}=(0,0.8,0.1), R_{y}^{*}=(0.2,0,0.3), R_{z}^{*}=(0.4,0.5,0)$, since

$$
\begin{gathered}
\mathcal{K}_{R^{*}}\left(R_{x}^{*}\right)=(0.4,0.2,0.5) \neq R_{x}, \mathcal{K}_{R^{*}}\left(R_{y}^{*}\right)=(0.8,1,0.7)=R_{y} \\
\mathcal{K}_{R^{*}}\left(R_{z}^{*}\right)=(0.6,0.5,0.7) \neq R_{z},
\end{gathered}
$$

Hence $R_{y}^{*} \in \tau_{\mathcal{K}_{R^{*}}}$ but $R_{x}^{*}, R_{z}^{*} \notin \tau_{\mathcal{K}_{R^{*}}}$ and $\bigwedge_{z \in X}(R(z, c) \rightarrow R(z, b))=0.3 \neq$ $R(c, b)=0.5$ from:

$$
\left(\bigwedge_{z \in X}(R(z, x) \rightarrow R(z, y))\right)=\left(\begin{array}{ccc}
1 & 0.2 & 0.9 \\
0.8 & 1 & 0.7 \\
0.6 & 0.3 & 1
\end{array}\right)
$$

(3) Put $R^{2}(x, y)=\bigvee_{z \in X}(R(x, z) \odot R(z, y))$, we obtain a relation $R^{2}$ as

$$
R^{2}=\left(\begin{array}{ccc}
1 & 0.4 & 0.9 \\
0.8 & 1 & 0.7 \\
0.6 & 0.5 & 1
\end{array}\right)
$$

Since $R^{2}(x, y) \odot R^{2}(y, z) \leq R^{2}(x, z)$ and $R^{2}(x, x)=1$ for all $x, y, z \in X, R^{2}$ is an $L$-fuzzy preorder. By Theorems 7(2) and 8(2), we have

$$
\begin{gathered}
\mathcal{K}_{R^{2 *}}(A)=\mathcal{K}_{R^{2 *}}\left(\mathcal{K}_{R^{2 *}}^{*}(A)\right), \\
\tau_{\mathcal{K}_{R^{2 *}}}=\left\{\bigvee_{x \in X}\left(A(x) \odot R^{2}(x,-)\right) \mid A \in L^{X}\right\}
\end{gathered}
$$


Since

$$
\begin{aligned}
R_{\tau_{\mathcal{K}_{R^{2 *}}}}(x, y) & =\bigwedge_{A \in L^{X}}\left(\bigvee_{z \in X}\left(A(z) \odot R^{2}(z, x)\right) \rightarrow \bigvee_{w \in X}\left(A(w) \odot R^{2}(w, y)\right)\right) \\
& \geq \bigwedge_{A \in L^{X}}\left(\bigwedge_{z \in X}\left(\left(A(z) \odot R^{2}(z, x)\right) \rightarrow\left(A(z) \odot R^{2}(z, y)\right)\right)\right. \\
& \geq R^{2}(x, y), \\
R_{\tau_{\mathcal{K}_{R^{2 *}}}}(x, y) & =\bigwedge_{A \in L^{X}}\left(\bigvee_{z \in X}\left(A(z) \odot R^{2}(z, x)\right) \rightarrow \bigvee_{w \in X}\left(A(w) \odot R^{2}(w, y)\right)\right) \\
& \leq \bigwedge_{p \in X}\left(\bigvee_{z \in X}\left(\top_{p}(z) \odot R^{2}(z, x)\right) \rightarrow \bigvee_{w \in X}\left(\top_{p}(w) \odot R^{2}(w, y)\right)\right) \\
& \leq \bigwedge_{p \in X}\left(R^{2}(p, x) \rightarrow R^{2}(p, y)\right) \leq R^{2}(x, x) \rightarrow R^{2}(x, y)=R^{2}(x, y),
\end{aligned}
$$

we have $R^{2}(x, y)=R_{\tau_{\mathcal{K}^{2 *}}}$.

For $R_{x}^{2}=(1,0.4,0.9), R_{y}^{2}=(0.8,1,0.7), R_{z}^{2}=(0.6,0.5,1)$, since

$$
\begin{gathered}
\mathcal{K}_{R^{2 *}}\left(R_{x}^{2}\right)=(0,0.6,0.1)=R_{x}^{2 *}, \quad \mathcal{K}_{R^{2 *}}\left(R_{y}^{2}\right)=(0.2,0,0.3)=R_{y}^{2 *} \\
\mathcal{K}_{R^{2 *}}\left(R_{z}^{2}\right)=(0.4,0.5,0)=R_{z}^{2 *}
\end{gathered}
$$

Hence $R_{x}^{2}, R_{y}^{2}, R_{z}^{2} \in \tau_{\mathcal{K}_{R^{2 *}}}$ and $\bigwedge_{z \in X}\left(R^{2}(z, a) \rightarrow R^{2}(z, b)\right)=R^{2}(a, b)$ for all $a, b \in X$.

(4) Put $R^{[2]}(x, y)=\bigvee_{z \in X}(R(x, z) \odot R(y, z))$, we obtain an $L$-fuzzy relation $R^{[2]}$ as

$$
R^{[2]}=\left(\begin{array}{ccc}
1 & 0.8 & 0.9 \\
0.8 & 1 & 0.7 \\
0.9 & 0.7 & 1
\end{array}\right)
$$

Since $R^{[2]}(x, z) \odot R^{[2]}(y, z) \leq R^{[2]}(x, y)$ and $R^{[2]}(x, x)=1$ for all $x, y, z \in X$, $R^{[2]}$ is reflexive and Euclidean. By Theorems 7(2) and 8(2), we have

$$
\begin{gathered}
\mathcal{K}_{R^{[2] *}}^{*}(A)=\mathcal{K}_{R^{[2] *}}\left(\mathcal{K}_{R^{[2] *}}(A)\right), \\
\tau_{\mathcal{K}_{R^{[2] *}}}=\left\{\bigwedge_{x \in X}\left(A(x) \rightarrow R^{[2] *}(x,-)\right) \mid A \in L^{X}\right\} .
\end{gathered}
$$


Since

$$
\begin{aligned}
R_{\tau_{\mathcal{K}_{R}}[2] *}(x, y)= & \bigwedge_{A \in L^{X}}\left(\bigwedge_{z \in X}\left(A(z) \rightarrow R^{[2] *}(z, x)\right)\right. \\
& \left.\rightarrow \bigwedge_{w \in X}\left(A(w) \rightarrow R^{[2] *}(w, y)\right)\right) \\
\geq & \bigwedge_{A \in L^{X}}\left(\bigwedge _ { z \in X } \left(\left(A(x) \rightarrow R^{[2] *}(z, x)\right)\right.\right. \\
& \left.\rightarrow\left(A(z) \rightarrow R^{[2]}(z, y)\right)\right) \\
\geq & \bigwedge_{z \in X}\left(R^{[2] *}(z, x) \rightarrow R^{[2] *}(z, y)\right)=\bigwedge_{z \in X}\left(R^{[2]}(z, y)\right. \\
& \left.\rightarrow R^{[2]}(z, x)\right) \\
\geq & R^{[2]}(y, x)=R^{[2]-1}(x, y), \\
= & \bigwedge_{A \in L^{X}}\left(\bigwedge_{z \in X}\left(A(z) \rightarrow R^{[2] *}(z, x)\right)\right. \\
& \left.\rightarrow \bigwedge_{w \in X}\left(A(w) \rightarrow R^{[2] *}(w, y)\right)\right) \\
R_{\tau_{\mathcal{K}}[2] *}(x, y) & \bigwedge_{p \in X}\left(\bigwedge _ { z \in X } \left(\top_{p}(z)\right.\right. \\
& \left.\left.\rightarrow R^{[2] *}(z, x)\right) \rightarrow \bigvee_{w \in X}\left(\top_{p}(w) \rightarrow R^{[2] *}(w, y)\right)\right) \\
\leq & \bigwedge_{p \in X}\left(R^{[2] *}(p, x) \rightarrow R^{[2] *}(p, y)\right) \\
= & \bigwedge_{p \in X}\left(R^{[2]}(p, y) \rightarrow R^{[2]}(p, x)\right) \leq R^{[2]}(y, x)=R^{[2]-1}(x, y),
\end{aligned}
$$

we have $R^{[2]-1}(x, y)=R_{\tau_{\mathcal{K}}(2) *}$.

For $R_{x}^{[2] *}=(0,0.2,0.1), R_{y}^{[2] *}=(0.2,0,0.3), R_{z}^{[2] *}=(0.1,0.3,0)$, since

$$
\begin{gathered}
\mathcal{K}_{R^{[2] *}}\left(R_{x}^{[2] *}\right)=(1,0.8,0.9)=R_{x}^{[2]}, \quad \mathcal{K}_{R^{[2] *}}\left(R_{y}^{[2] *}\right)=(0.8,1,0.7)=R_{y}^{[2]} \\
\mathcal{K}_{R^{[2] *}}\left(R_{z}^{[2] *}\right)=(0.9,0.7,1)=R_{z}^{[2]}
\end{gathered}
$$

Hence $R_{x}^{[2] *}, R_{y}^{[2] *}, R_{z}^{[2] *} \in \tau_{\mathcal{K}_{R}[2] *}$ and $\bigwedge_{z \in X}\left(R^{[2]}(z, a) \rightarrow R^{[2]}(z, b)\right)=R^{[2]}(a, b)$ for all $a, b \in X$.

\section{References}

[1] R. Bělohlávek,Fuzzy Relational Systems, Kluwer Academic Publishers, New York , (2002).

[2] P. Hájek, Metamathematices of Fuzzy Logic, Kluwer Academic Publishers, Dordrecht (1998).

[3] Y.C. Kim, L-join meet approximation operators to appear International Journal of Pure and Applied Mathematics.

[4] Y.C. Kim, Alexandrov L-topologies, to appear International Journal of Pure and Applied Mathematics. 
[5] H. Lai, D. Zhang, Fuzzy preorder and fuzzy topology, Fuzzy Sets and Systems, 157 (2006), 1865-1885, doi: 10.1016/j.fss.2006.02.013.

[6] H. Lai, D. Zhang, Concept lattices of fuzzy contexts: Formal concept analysis vs. rough set theory, Int. J. Approx. Reasoning, 50 (2009), 695-707, doi: $10.1016 /$ j.ijar.2008.12.002.

[7] Z. Pawlak, Rough sets, Int. J. Comput. Inf. Sci., 11 (1982), 341-356.

[8] Z. Pawlak, Rough probability, Bull. Pol. Acad. Sci. Math., 32(1984), 607615.

[9] A. M. Radzikowska, E.E. Kerre, A comparative study of fuzy rough sets, Fuzzy Sets and Systems, 126(2002), 137-155, doi: 10.1016/so1650114(01)00032-x.

[10] Y.H. She, G.J. Wang, An axiomatic approach of fuzzy rough sets based on residuated lattices, Computers and Mathematics with Applications, $\mathbf{5 8}$ (2009), 189-201, doi: 10.1016/j.camwa.2009.03.100.

[11] Zhen Ming Ma, Bao Qing Hu, Topological and lattice structures of Lfuzzy rough set determined by lower and upper sets, Information Sciences, 218(2013), 194-204, doi: 10.1016/j.ins.2012.06.029. 
